\section{Validation of a new photogrammetric technique to monitor the treatment effect of Botulinum toxin in synkinesis}

NT Mabvuure' ${ }^{1,2}$, M-J Hallam¹, V Venables ${ }^{1}$ and C Nduka ${ }^{1}$
${ }^{1}$ Queen Victoria Hospital NHS Foundation Trust, East Grinstead, UK

${ }^{2}$ Brighton and Sussex Medical School, Brighton, UK

Correspondence: M-J Hallam, Queen Victoria Hospital NHS Foundation Trust, Holtye Road, East Grinstead, West Sussex RH19 3DZ, UK.

Tel: +44 (0)1342 414000; Fax: +44 (0)1342 414414 E-mail: mj_hallam@ hotmail.co.uk

This paper was presented at the Society of Academic and Research Surgery 2012 Meeting, Nottingham, England, 5 January 2012

Received: 7 November 2012 Accepted in revised form: 11 March 2013 Published online: 17 May 2013

\begin{abstract}
Aims To validate a new photogrammetric technique for quantifying eye surface area and using this to quantify the degree of improvement in symmetry in patients with oral-ocular synkinesis following Botulinum toxin injection.

Study design Feasibility study and retrospective outcomes analysis Methods Ten patients' photographs were chosen from a photographic database.

Their eye surface areas were measured independently by two raters using a graphics tablet. One rater repeated the procedure after 15 days. Bland-Altman plots were computed, ascertaining inter-rater and intra-rater variability. The eye surface areas of 19 patients were then derived from photographs taken before and after Botulinum toxin injections. Paired $t$-tests were used to analyse the significance of the difference in pre- and post-treatment symmetry.

Results Ninety per cent of eye surface areas derived from the two raters were within a coefficient of variation of 0.1 (95\% CI: $0.05-$ $0.15)$. Similarly, $90 \%$ of eye surface areas derived from one rater had a coefficient of variation of 0.08 (95\% CI: 0.04-0.12).

Botulinum toxin significantly reduced synkinesis resulting from lip puckering, Mona Lisa smiling and Hollywood smiling $(P<0.05)$.

Conclusions We have proposed a clinically valid tool for quantifying the effects of Botulinum toxin treatment for oral-ocular synkinesis. We recommend this method be used to monitor the response of such patients when receiving Botulinum toxin treatment.
\end{abstract}

Level of evidence $2 \mathrm{c}$.

Eye (2013) 27, 860-864; doi:10.1038/eye.2013.91; published online 17 May 2013

Keywords: synkinesis; botulinum toxin; facial palsy; photogrammetric

\section{Introduction}

Patients with facial nerve injuries are frequently afflicted by synkinesis, a movement disorder principally attributed to aberrant nerve regeneration. ${ }^{1}$ The incidence of synkinesis in patients recovering from facial paralysis is between $15-50 \%$ depending on the series. ${ }^{2,3}$ Synkinesis specifically refers to the involuntary contraction of a muscle or muscle group, in addition to that required for a desired expression or movement; for example, a patient's eye may involuntarily close when smiling due to inappropriate innervation of the obicularis oculi muscle. Synkinetic movements affecting facial muscle groups may not only result in the impairment of normal facial functions, such as eating or eye closing, but may also result in difficulties in conveying appropriate or desired facial expressions. Synkinesis, therefore, frequently has a negative effect on a patient's quality of life. ${ }^{4,5}$

Since the early 1990s, directed Botulinum toxin injection to affected muscles has been the mainstay of treatment of synkinetic movement disorders. ${ }^{6,7}$ The Botulinum toxin inhibits the release of acetylcholine from the presynaptic nerve terminal at the neuromuscular junction, preventing the propagation of the action potential and contraction of the targeted muscle. The resulting (temporary) 
paralysis of synkinetic muscles has been demonstrated to improve the quality of life in affected individuals. ${ }^{5}$ However, the optimal dosing range of Botulinum toxin for treating synkinesis has not yet been established. It is frequently administered on an ad hoc basis, exposing patients to the possibility of under- or overtreatment.

One of the problems faced when establishing an efficacious Botulinum toxin treatment dose is that there is no universally accepted technique within the literature for objectively measuring the effects of treatment. Studies have attempted to address this issue; one such study used patient-reported perceptions of improvement after treatment, ${ }^{8}$ and although the accuracy of such objective data cannot be guaranteed, the data is useful as it reflects patient satisfaction with treatment. ${ }^{9}$ Clinician-based scales such as the House-Brackmann, Facial Grading and the Sunnybrook systems are also widely used, but also are subjective and subject to wide inter-rater variability. ${ }^{10}$ Furthermore, these scales do not precisely quantify the degree of synkinesis present. Therefore, there has been a concerted effort to develop computer-based systems in order to provide objective measures of facial motion. ${ }^{11-16}$ Although largely improving the objectivity of facial assessment, the cost of these specialised systems likely limits the widespread uptake of these systems, especially in these fiscal times. Thus, there is a clear need to continue investigating alternative, cheaper and more reliable techniques.

Eye closure resulting from voluntary mouth movements (oral-ocular synkinesis) is the most common synkinetic movement resulting from volitional mouth movements. ${ }^{4}$ Techniques such as the Maximal Static Response assay have been used in attempts to quantify the degree of ocular-oral synkinesis, ${ }^{14}$ but no methods have quantified oral-ocular synkinesis enough to guarantee wide uptake. At our unit, patients are routinely photographed before and after Botulinum toxin treatment. From these photographs, improvements in symmetry and position of the peri-ocular tissues are subjectively obvious, but like other institutions, we have struggled to accurately quantify the clinically obvious response to treatment.

In the current study, we propose to validate a novel photogrammetric method of assessing the synkinetic peri-ocular region and to accurately quantify the effects of Botulinum toxin therapy in the treatment of oral-ocular synkinesis through direct comparisons before and after treatment.

\section{Materials and methods}

\section{Hardware and software}

All photographs included in the present study were taken using either a Nikon D700 or a Nikon D300 camera (both Nikon, Tokyo, Japan). The photographs included

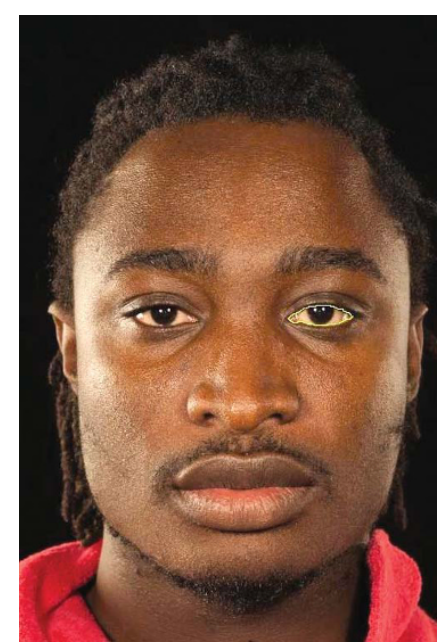

Figure 1 Example of a tracing line drawn to determine the eye surface area. The line starts at the endocanthion, and follows the border of the sclera and conjunctiva to the exocanthion around the eye.

were all frontal photographs taken with either a $2128 \times 1416$ pixel $($ D300) or a $1296 \times 1936$ pixel (D700) setting. For image analysis, ImageJ software (http:// rsbweb.nih.gov/ij/) was used. The perimeter of the eye was traced using a graphics tablet (Bamboo, Wacom, Vancouver, WA, USA) and the number of pixels covered by the perimeter derived from ImageJ. Starting at the endocanthion, a line was drawn following the border of the sclera and conjunctiva to the exocanthion and back to the endocanthion (Figure 1).

\section{Validation study}

For validation purposes, 10 subjects ( 5 male, 5 female) were randomly selected from the hospital's database of facial photographs. Two raters (NTM and MJH) independently measured the area of the right and left eyes for each subject to derive inter-rater variability of the measurements. Additionally, NTM repeated the measurements on the same subjects after 15 days, without referring to the record of the first measurements, to derive intra-rater variability.

\section{Photogrammetric analysis}

A database of 246 patients, treated by the senior author for facial palsy at a tertiary referral centre between 2007 and 2011, was retrospectively searched for patients with oral-ocular synkinesis and a full set of standardised photos taken in a studio before and after Botulinum toxin treatment by a professional medical photographer. Nineteen suitable candidates (16 female, 3 male) were retrospectively identified. A full set of standardised photos was taken to mean photographs taken with the 
Nikon D700/D300 with either a $105 \mathrm{~mm}$ lens (FX format) or $60 \mathrm{~mm}$ lens (DX format) at $1 / 160 \mathrm{~s}$ and $\mathrm{f} 22$, under constant lighting using four Bowens lights (Bowens, Clacton on Sea, UK, two backlights and two front lights), on a black background, demonstrating the following poses at maximal intensity under and distance: repose, smiling with (canine/ Hollywood smile) and without showing teeth (Mona Lisa smile), ${ }^{17}$ lip puckering, blowing out cheeks and showing the lower teeth. All images were stored on the hospital's secure online database where they are kept for a period of 10 years before deletion. For each patient, the specific volitional movement(s) resulting in eye closure was recorded. The ratio of the area of the synkinetic eye to that of the normal eye, during specific volitional movements, was calculated both before and after treatment.

\section{Statistical analysis}

Bland-Altman plots ${ }^{18}$ were constructed to illustrate trends in the area measurements and to visually depict inter- and intra-rater repeatability of our technique. Repeatability coefficients and 95\% confidence intervals thereof were also determined. For all photogrammetric analyses, ratios of the area of the synkinetic eye to that of the normal eye were used. The difference in the pre- and post-treatment ratios for each patient were analysed using paired $t$-tests, with significance regarded as $P<0.05$.

\section{Results}

\section{Inter-rater reproducibility}

Table 1 shows the areas of the eyes as measured by the two raters independently. The mean difference between the ratios derived from the two raters was 0.03 . The Bland-Altman graph for these differences (Figure 2a) shows that $90 \%$ of the ratios of eye areas derived from measurements made by the two independent raters were acceptably similar. The coefficient of the inter-rater variation was 0.1 (95\% CI: $0.05-0.15)$.

\section{Intra-rater repeatability}

Table 2 shows the areas of the eyes as measured by NTM at two time points15 days apart. The mean difference between the ratios derived at the two different times was also 0.03 . The Bland-Altman graph for these differences (Figure $2 \mathrm{~b}$ ) shows that $90 \%$ of the ratios of eye areas derived from measurements made by NTM 15 days apart were acceptably similar. The coefficient of variation for test-retest reliability was 0.08 (95\% CI: 0.04-0.12).

\section{Photogrammetric analysis}

Botulinum toxin injection did not result in a statistically significant difference in the symmetry of eyes before and
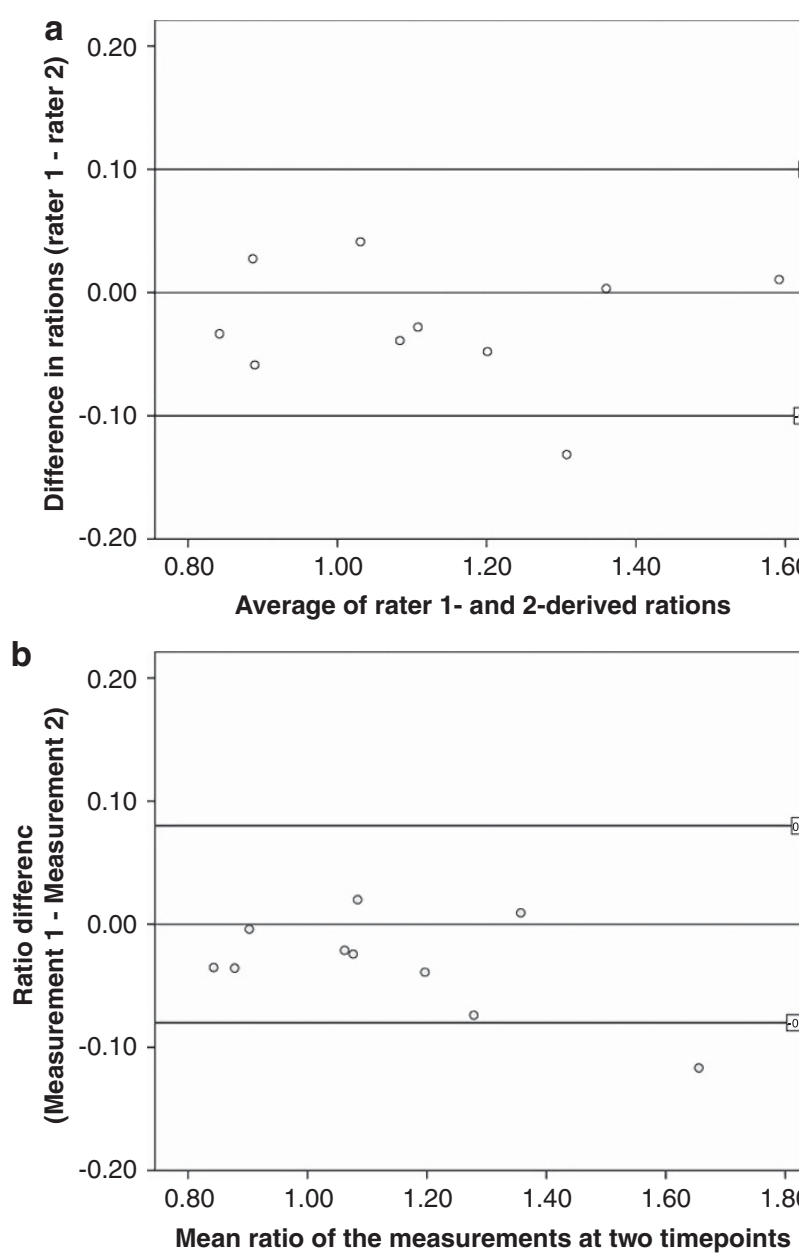

Figure 2 (a) Bland-Altman plot of the inter-rater variation in eye surface area measurements. Ninety per cent $(9 / 10)$ of the areas measured by the two raters are within the coefficient of variation limits of \pm 0.1 . (b) Bland-Altman plot of the test-retest reliability in measurements. Ninety per cent $(9 / 10)$ of the areas measured by one rater were within the coefficient of variation limits of \pm 0.08 .

after treatment where patients were in repose $(P=0.35$; Table 3 ). Where Botulinum toxin was injected in patients with eye closure resulting from lip puckering, Mona Lisa smiling and Hollywood smiling, a statistically significant improvement in symmetry was seen $(P<0.05$; Table 3$)$. Symmetry was not statistically significantly improved following injection in patient with synkinesis resulting from blowing out cheeks and using lip depressors to show lower teeth $(P=0.08$ and 0.07 , respectively; Table 3$)$.

\section{Discussion}

The first aim of this study was to validate the use of a graphics tablet and ImageJ to calculate the area of the eyes. The limits of agreement set for the Bland-Altman plot (Figure 2a) showed that the two independent raters 
Table 1 Eye surface areas derived by two independent raters

\begin{tabular}{|c|c|c|c|c|c|c|c|}
\hline \multirow[t]{3}{*}{ Subject } & \multicolumn{3}{|c|}{ Rater 1} & \multicolumn{3}{|c|}{ Rater 2} & \multirow{3}{*}{$\begin{array}{c}\text { Ratio } \\
\text { difference }\end{array}$} \\
\hline & \multicolumn{2}{|c|}{ Area (pixels) } & \multirow[b]{2}{*}{$\begin{array}{l}\text { Ratio } \\
(R: L)\end{array}$} & \multicolumn{2}{|c|}{ Area (pixels) } & \multirow[b]{2}{*}{$\begin{array}{l}\text { Ratio } \\
(R: L)\end{array}$} & \\
\hline & $R$ & $L$ & & $R$ & $L$ & & \\
\hline 1 & 7105 & 6495 & 1.09 & 7353 & 6554 & 1.12 & -0.03 \\
\hline 2 & 8295 & 9211 & 0.90 & 8158 & 9343 & 0.87 & 0.03 \\
\hline 3 & 12734 & 9351 & 1.36 & 12606 & 9279 & 1.36 & 0.00 \\
\hline 4 & 142451 & 13548 & 1.05 & 138981 & 13757 & 1.01 & 0.04 \\
\hline 5 & 5304 & 6167 & 0.86 & 5712 & 6217 & 0.92 & -0.06 \\
\hline 6 & 9252 & 7860 & 1.18 & 9279 & 7575 & 1.22 & -0.04 \\
\hline 7 & 5860 & 7100 & 0.83 & 6064 & 7061 & 0.86 & -0.03 \\
\hline 8 & 14509 & 9085 & 1.60 & 14269 & 8994 & 1.59 & 0.01 \\
\hline 9 & 11411 & 9191 & 1.24 & 11754 & 8561 & 1.37 & -0.13 \\
\hline 10 & 10003 & 9398 & 1.06 & 10144 & 9194 & 1.10 & -0.04 \\
\hline
\end{tabular}

Abbreviations: R, right; L, left.

scored within two scale points of each other on $90 \%$ of measurements. There was no systematic bias between the raters. A coefficient of variation of 0.1 means that two ratios derived from area measurements made by two different raters are likely to differ by at most 0.1 . This difference is small enough to make the level of inter-rater variation clinically acceptable and useful. Similarly, clinically acceptable test-retest reliability was demonstrated (Figure $2 b$ ), supported by a coefficient of variation of 0.08 . Figure $2 b$ also shows that the majority of the second area measurements are slightly smaller than the first measurements perhaps due to the rater becoming more comfortable with using the graphics tablet. These results show that this technique is a reliable method of measuring the area of an eye on a patient photograph.

Aside from its reliability, this method is also costeffective. Clinicians in the developed world, certainly in UK, all have access to computers and the internet, from which ImageJ can be downloaded free of charge. Therefore, the only new hardware to be bought when using this method is a graphics tablet, although it is hoped that the photography departments of most hospitals will have these. Indeed, it is certainly possible to trace the borders of the eyes using a normal computer mouse. However, our experience has shown that this task is much simplified and expedited by using a graphics tablet that can be purchased for $\sim £ 50$.

The second part of this study aimed to perform a photogrammetric analysis on the images of patients treated with Botulinum toxin for oral-ocular synkinesis, using the technique validated above. Botulinum toxin treatment did not make a statistically significant difference to the symmetry of patients' eyes in repose (Table 1). This is to be expected as synkinesis, by definition, is produced following a volitional movement, and therefore, reasonable symmetry will normally be
Table 2 Eye surface areas as measured by one rater 15 days apart

\begin{tabular}{|c|c|c|c|c|c|c|c|}
\hline \multirow[t]{3}{*}{ Subject } & \multicolumn{3}{|c|}{ First measurement } & \multicolumn{3}{|c|}{ Second measurement } & \multirow{3}{*}{$\begin{array}{c}\text { Ratio } \\
\text { difference }\end{array}$} \\
\hline & \multicolumn{2}{|c|}{ Area (pixels) } & \multirow[b]{2}{*}{$\begin{array}{l}\text { Ratio } \\
(R: L)\end{array}$} & \multicolumn{2}{|c|}{ Area (pixels) } & \multirow[b]{2}{*}{$\begin{array}{l}\text { Ratio } \\
(R: L)\end{array}$} & \\
\hline & $R$ & $L$ & & $R$ & $L$ & & \\
\hline 1 & 7105 & 6495 & 1.09 & 7501 & 6984 & 1.07 & 0.02 \\
\hline 2 & 8295 & 9211 & 0.90 & 8375 & 9259 & 0.90 & 0.00 \\
\hline 3 & 12734 & 9351 & 1.36 & 13034 & 9637 & 1.35 & 0.01 \\
\hline 4 & 14245 & 13548 & 1.05 & 14280 & 13313 & 1.07 & -0.02 \\
\hline 5 & 5304 & 6167 & 0.86 & 5463 & 6099 & 0.90 & -0.04 \\
\hline 6 & 9252 & 7860 & 1.18 & 9293 & 7642 & 1.22 & -0.04 \\
\hline 7 & 5860 & 7100 & 0.83 & 5842 & 6789 & 0.86 & -0.03 \\
\hline 8 & 14509 & 9085 & 1.60 & 15180 & 8858 & 1.71 & -0.11 \\
\hline 9 & 11411 & 9191 & 1.24 & 11639 & 8848 & 1.32 & -0.08 \\
\hline 10 & 10003 & 9398 & 1.06 & 9647 & 8862 & 1.09 & -0.03 \\
\hline
\end{tabular}

Abbreviations: R, right; L, left.

Table 3 Eye surface area ratios before and after Botulinum toxin treatment

\begin{tabular}{lcccc}
\hline $\begin{array}{l}\text { Volitional } \\
\text { movement }\end{array}$ & $\begin{array}{c}\text { Number of } \\
\text { patients }\end{array}$ & $\begin{array}{c}\text { Prebotox s: } n \\
\text { ratio (mean) }\end{array}$ & $\begin{array}{c}\text { Postbotox s: } n \\
\text { ratio (mean) }\end{array}$ & P-value \\
\hline $\begin{array}{l}\text { Repose } \\
\text { Lip pucker }\end{array}$ & 19 & 0.932322109 & 0.959508251 & 0.35 \\
$\begin{array}{l}\text { Mona Lisa } \\
\text { smile }\end{array}$ & 15 & 0.48819484 & 0.761099614 & 0.001 \\
$\begin{array}{l}\text { Canine/ } \\
\begin{array}{l}\text { Hollywood } \\
\text { smile }\end{array}\end{array}$ & 15 & 0.615228332 & 0.819504053 & 0.01 \\
$\begin{array}{l}\text { Blowing out } \\
\text { cheeks }\end{array}$ & 17 & 0.484640603 & 0.577822105 & 0.08 \\
$\begin{array}{l}\text { Showing } \\
\text { bottom teeth }\end{array}$ & 17 & 0.612998249 & 0.761525562 & 0.07 \\
\hline
\end{tabular}

Abbreviation: $\mathrm{s}$ : n,ratio of area of synkinetic eye (s) to the normal eye (n).

seen in repose. The effect of Botulinum toxin treatment of ocular synkinesis resulting from the volitional oral movements of blowing out the cheeks and using the lip depressors to show the bottom teeth approached statistical significance, $P=0.08$ and 0.07 , respectively. However, because the mean eye area ratios before and after treatment clearly show increased symmetry (Table 3), that is, the post-treatment ratio is closer to 1.0 than the pre-treatment ratio, it is likely that the marginal statistical insignificance would be rectified in a larger cohort. Also, while statistical significance may not have been achieved within this group, it is still useful for a clinician to know the eye area ratios pre- and post-treatment to monitor individual patient responses, to allow personalised tailoring of therapy. One may also infer this result to suggest that the treatment dose used in these cases was sub-optimal and further treatments may 
require a higher concentration of Botulinum toxin. One reason for this is that higher toxin doses were likely to cause lagopthalmos in some patients; therefore, the objective of treatment needed to be balanced by the desire to avoid adverse effects. We suggest, therefore, that our method is also a useful way of monitoring response to and directing future treatments.

Statistically significant improvements in symmetry following treatment were seen, however, with synkinesis resulting from lip puckering, smiling with lips closed (Mona Lisa smile) and smiling with teeth showing (Table 3). These results quantify the physical effect of Botulinum toxin, which other authors have previously shown to result in an improvement in patients' quality of life. ${ }^{5}$ This quantification will be useful if comparisons of the efficacy of different Botulinum toxin preparations were to be made. However, a limitation of this method is that it is specific for quantifying oral-ocular synkinesis and cannot in its present form be used to quantify other types of facial synkinesis. We are currently conducting further research in this area in order to develop similar tools that may be used to assess other synkinetic movements with accuracy.

\section{Conclusions}

This paper establishes the validity of a new photogrammetric tool that can be used to monitor the treatment effect of Botulinum toxin in patients with oral-ocular synkinesis. We therefore recommend this method be used to monitor patients with oral-ocular synkinesis who are to receive Botulinum toxin treatment.

\section{Summary}

What was known before

- Synkinesis is a common problem in facial palsy. Botulinum toxin is frequently used for treatment. Treatment response is difficult to quantify.

\section{What this study adds}

- New photogrammetric technique for quantifying eye surface area. A method to quantify the degree of improvement in symmetry in patients with oral-ocular synkinesis following Botulinum toxin injection.

\section{Conflict of interest}

The authors declare no conflict of interest.

\section{Acknowledgements}

We thank Stephanie Goubet and Liz Cheek at Brighton and Sussex Medical School for statistical advice.

\section{References}

1 Husseman J, Mehta RP. Management of synkinesis. Facial Plast Surg 2008; 24(2): 242-249.

2 Celik M, Forta H, Vural C. The development of synkinesis after facial nerve paralysis. Eur Neurol 2000; 43(3): 147-151.

3 Yamamoto E, Nishimura H, Hirono Y. Occurrence of sequelae in Bell's palsy. Acta Otolaryngol Suppl 1988; 446: 93-96.

4 Beurskens $\mathrm{CH}$, Oosterhof J, Nijhuis-van der Sanden MW. Frequency and location of synkineses in patients with peripheral facial nerve paresis. Otol Neurotol 2010; 31(4): 671-675.

5 Mehta RP, Hadlock TA. Botulinum toxin and quality of life in patients with facial paralysis. Arch Facial Plast Surg 2008; 10(2): 84-87.

6 Chong PN, Ong B, Chan R. Botulinum toxin in the treatment of facial dyskinesias. Ann Acad Med Singapore 1991; 20(2): 223-227.

7 Roggenkamper P, Laskawi R, Damenz W, Schroder M, Nussgens Z. Botulinum toxin treatment of synkinesia following facial paralysis. HNO 1990; 38(8): 295-297.

8 Borodic G, Bartley M, Slattery W, Glasscock M, Johnson E, Malazio $\mathrm{C}$ et al. Botulinum toxin for aberrant facial nerve regeneration: double-blind, placebo-controlled trial using subjective endpoints. Plast Reconstr Surg 2005; 116(1): 36-43.

9 Rogers CR, Schmidt KL, VanSwearingen JM, Cohn JF, Wachtman GS, Manders EK et al. Automated facial image analysis: detecting improvement in abnormal facial movement after treatment with botulinum toxin A. Ann Plast Surg 2007; 58(1): 39-47.

10 de Ru JA, Braunius WW, van Benthem PPG, Busschers WB, Hordijk GJ. Grading facial nerve function: why a new grading system, the MoReSS, should be proposed. Otol Neurotol 2006; 27(7): 1030-1036.

11 Hontanilla B, Auba C. Automatic three-dimensional quantitative analysis for evaluation of facial movement. J Plast Reconstr Aesthet Surg 2008; 61(1): 18-30.

12 Linstrom CJ. Objective facial motion analysis in patients with facial nerve dysfunction. Laryngoscope 2002; 112(7 Pt 1): 1129-1147.

13 Coulson SE, Croxson GR, Gilleard WL. Quantification of the three-dimensional displacement of normal facial movement. Ann Otol Rhinol Laryngol 2000; 109(5): 478-483.

14 Bajaj-Luthra A, VanSwearingen J, Thornton RH, Johnson PC. Quantitation of patterns of facial movement in patients with ocular to oral synkinesis. Plast Reconstr Surg 1998; 101(6): 1473-1480.

15 Neely JG, Joaquin AH, Kohn LA, Cheung JY. Quantitative assessment of the variation within grades of facial paralysis. Laryngoscope 1996; 106(4): 438-442.

16 Neely JG, Cheung JY, Wood M, Byers J, Rogerson A. Computerized quantitative dynamic analysis of facial motion in the paralyzed and synkinetic face. Am J Otol 1992; 13(2): 97-107.

17 Sawyer AR, See M, Nduka C. Quantitative analysis of normal smile with 3D stereophotogrammetry-an aid to facial reanimation. J Plast Reconstr Aesthet Surg 2010; 63(1): 65-72.

18 Bland JM, Altman DG. Statistical methods for assessing agreement between two methods of clinical measurement. Lancet 1986; 1(8476): 307-310. 